Acta Crystallographica Section A

Foundations of

Crystallography

ISSN 0108-7673

Received 19 August 2005

Accepted 12 January 2006

(C) 2006 International Union of Crystallography

Printed in Great Britain - all rights reserved

\section{Application of modern tensor calculus to engineered domain structures. 2. Tensor distinction of domain states}

\author{
Vojtěch Kopský \\ Institut of Physics, Czech Academy of Sciences, Na Slovance 2, 18221 Praha 8, Czech Republic, \\ and Department of Mathematics and Didactics of Mathematics, Pedagogical Faculty, Technical \\ University of Liberec, Hálkova 6, 46117 Liberec 1, Czech Republic. Correspondence e-mail: \\ kopsky@fzu.cz
}

\begin{abstract}
The theory of domain states is reviewed as a prerequisite for consideration of tensorial distinction of domain states. It is then shown that the parameters of the first domain in a ferroic phase transition from a set of isomorphic groups of the same oriented Laue class can be systematically and suitably represented in terms of typical variables. On replacing these variables by actual tensor components according to the previous paper [Kopský (2006), Acta Cryst. A62, 47-64], we can reveal the tensorial parameters associated with each particular symmetry descent. Parameters are distinguished by the ireps to which they belong and this can be used to determine which of them are the principal parameters that distinguish all domain states, in contrast to secondary parameters which are common to several domain states. In general, the parameters are expressed as the covariant components of the tensors. A general procedure is described which is designed to transform the results to Cartesian components. It consists of two parts: the first, called the labelling of covariants, and its inverse, called the conversion equations. Transformation of parameters from the first domain state to other states is now reduced to irreducible subspaces whose maximal dimension is three in contrast with higher dimensions of tensor spaces. With this method, we can explicitly calculate tensor parameters for all domain states. To find the distinction of pairs of domain states, it is suitable to use the concept of the twinning group which is briefly described.
\end{abstract}

\section{Ferroic phase transitions}

The structure of a crystal depends frequently on external isotropic conditions, which means the temperature $T$ and the isotropic (hydrostatic) pressure $p$. For example, the structure of ice is described by a phase diagram which contains regions of $(T, p)$ corresponding to 11 different structures. When the external conditions change across the border between two phases in the diagram, the structure also changes. This phenomenon is called a structural phase transition and it is based on the model introduced by Landau (see collection of papers by Landau, 1937). This model assumes a certain kind of instability which causes the atoms to change their positions. The change of temperature and pressure always leads to changes of the structure - the lattice parameters and distances between atoms change within certain limits without a change of the structure type and hence without a change of the spacegroup-symmetry type. A structural phase transition caused by such displacements of atoms due to a change in symmetry are called displacive. The physical cause of the transition is a certain mode, whose eigenfrequency tends to zero on crossing a certain line of the $(T, p)$ diagram. This mode is called a soft mode and we used to say that this mode freezes, causing permanent distortions and a change of symmetry.

The importance of the theory of group representations in various descriptions of this phenomenon was realized early on by Landau although he was evidently not aware of the state of the art of group theory in 1937; he used his own term races of functions for the bases of irreducible representations. One of the best descriptions of group-theoretical considerations of structural phase transitions is given by Lyubarskii (1960); the use of density functions is more general than the usual model of displacive transitions. The components of distortions which cause the transition are called the transition parameter; the alternative name soft mode reflects the physical origin of the transition. The eigenenergy of this mode tends to zero as the isotropic conditions approach the transition point. The onset of this parameter may induce the onset of other changes described as secondary (improper, faint) parameters.

By ferroic phase transitions, we understand transitions in which the point symmetry of the crystal changes. This occurs in the majority of cases but there also exist transitions to 
equiclass subgroups in which the point symmetry does not change. Since the point symmetry decreases in ferroic transitions, the transitions are accompanied by an onset of tensor properties which were originally disallowed. The symmetry group $G$ of the original phase is called the parent group, the symmetry $F_{i}$ of the ferroic phase its ferroic subgroup. We shall talk about the symmetry descent $G \Downarrow\left\{F_{i}\right\}$ which characterizes the ferroic transition and our concern will be the qualitative change of tensor properties. However, neither the symmetry nor the newly acquired tensor properties are defined uniquely. If the ferroic group $F_{i}$ is not a normal subgroup, then its conjugate subgroups are equivalent and any of them may appear as the resulting symmetry. This shows that there does not exist just one specific ferroic state but a set of equivalent ferroic states. The crystal may therefore adopt one of these states but it may also happen that different states correspond to different regions of a crystal. Such regions are called ferroic domains and the states are called ferroic domain states. From now on we shall drop the adjective ferroic. The equivalence of domain states means that they have the same properties with reference to different Cartesian systems so that these properties manifest themselves in different ways with reference to a common Cartesian system of coordinates. A clear theory of domain states has been introduced by Janovec (1972), and been refined by Kopský $(1982 a, b, c, d)$.

The existence of domains has an important influence on the final properties of the crystal which is now being utilized in the developing technique of domain engineering. A multidomain sample may be considered as a special case of a polycrystalline state. In both cases, the sample consists of regions of the same structure oriented in different ways with reference to a common Cartesian coordinate system. However, the grains of a polycrystal may be oriented in a rather arbitrary manner while the possible mutual orientations of domains in a multidomain sample are subject to strict rules of the theory of domain states. There are two ways in which the domains affect the resulting properties of a multidomain structure: (i) the volume and spatial arrangement of domains; (ii) the domain walls in which the properties change from one state to another.

It is therefore of interest to develop methods for the calculation of the properties of individual domain states and for comparison of these properties in adjoining domains. The latter is usually called the tensorial distinction of domain states. The standard technique of the characters of ireps allows one to calculate the numbers of tensor components in which domain states differ (Janovec et al., 1992, 1993, 1994, 1995). Our concern is to describe a group-theoretical technique for the calculation of explicit tensor properties of individual domains. This calls for the introduction of some useful concepts.

\section{Stability spaces, strata and epikernels}

Stability spaces: Anticipating the use of group-theoretical concepts for the description of domain states in ferroic transitions, we first explain the relationship between subgroups and carrier spaces of ireps of the original parent group $G$. Let us consider a tensor space $V^{(A)}$. It contains subspaces $V^{(A)}(G)$ and $V^{(A)}\left(\left\{F_{i}\right\}\right)$ of tensors which are invariant under the groups $G$ and $F_{i}$, respectively. Such spaces are called the stability spaces of groups $G$ and $F_{i}$. The subspace $V^{(A)}(G)$ is described by tensorial invariants of the group $G$, while the complementary subspace is defined by components of tensorial $D_{R}^{(\alpha)}(G)$ covariants. In considering the symmetry descent $G \Downarrow\left\{F_{i}\right\}$, we have to find those covariant components that became invariant under the action of ferroic groups $F_{i}$. Although generally there may exist no such components for a given tensor $\mathbf{A}$, there always exist tensors for which such covariant components exist and hence the stability spaces $V^{(A)}\left(F_{i}\right)$ always contain the stability space $V^{(A)}(G)$ and, for some tensors, these subspaces are proper subspaces. From the set of conjugate subgroups $\left\{F_{i}\right\}$, we choose one subgroup $F_{1}$; the stability space of a conjugate subgroup $F_{i}=g_{i} F_{1} g_{i}^{-1}$ is related to the stability space $V^{(A)}\left(F_{1}\right)$ by $V^{(A)}\left(F_{i}\right)=g_{i} V^{(A)}\left(F_{1}\right)$. To avoid the necessity of calculation of these spaces for each particular tensor, we use the approach via typical variables and typical carrier spaces. The natural choice of matrix ireps and standard variables for groups of the same oriented Laue class, described in Kopský (2006b, hereafter referred to as paper 1), proves also to be the most intelligent and effective.

The typical carrier space $V_{o}(G)=\oplus_{\alpha} V_{\alpha}(G)$ of the parent group $G$ is defined as a direct sum of carrier spaces $V_{\alpha}(G)$ of $R$-ireps of the group $G$ with bases $\left\{\mathbf{e}_{\alpha, 1}, \mathbf{e}_{\alpha, 2}, \ldots, \mathbf{e}_{\alpha, d_{\alpha}}\right\}$. All invariants of the group $G$ are represented by a single vector $\mathbf{e}_{1}$ or by a single variable $\mathrm{x}_{1}$. All $D^{(\alpha)}(G)$ covariants are represented by a typical $D^{(\alpha)}(G)$ covariant $\left(x_{\alpha, 1}, x_{\alpha, 2}, \ldots, x_{\alpha, d_{\alpha}}\right)$.

Let us now consider a subgroup $F_{1}$ of the group $G$ which is potentially the symmetry of some ferroic state. To this subgroup there corresponds a subspace $V_{o}\left(F_{1}\right)=\oplus_{\alpha} V_{\alpha}\left(F_{1}\right)$ of the typical space $V_{o}$ which contains all those vectors of $V_{o}$ which are invariant under the action of the subgroup $F_{1}$. This space is called the typical stability space of the subgroup $F_{1}$, while individual $V_{\alpha}\left(F_{1}\right)$ are the typical stability spaces of $F_{1}$ in the carrier spaces $V_{\alpha}(G)$ of individual ireps of the group $G$. Expressing the stability spaces in terms of standard variables, we obtain the set of invariants of the group $F_{1}$ expressed as linear combinations of these variables. Comparing them with tensorial covariants, we obtain immediately those linear combinations of tensor components that onset at the phase transition from the parent group $G$ to the ferroic subgroup $F_{1}$. More than that, we can also identify the ireps of the parent group $G$ to which the onsetting parameters belong. Here we simply use the fact that subspaces $V_{\alpha a}^{(A)}(G)$ behave under the action of the group $G$ like copies of the typical spaces $V_{\alpha}(G)$.

Stabilizers and orbits: Let us now consider the action of the group $G$ on a set $\mathcal{S}$. The elements of $\mathcal{S}$ are called points and are denoted by $\mathrm{S}$. The action of $G$ assigns to each element $g \in G$ and to each point $S \in \mathcal{S}$ a point $g S \in \mathcal{S}$. Let us pick up a certain point $S$. Some elements $f \in G$ may leave the point $S$ invariant, so that $f \mathrm{~S}=\mathrm{S}$. It is easy to show that all elements of $G$ that leave the point invariant must constitute a group, which is called the stabilizer of the point $S$ under the action of $G$.

We denote this group by $F_{1}$ and the point by $\mathrm{S}_{1}$. Performing coset resolution, 


$$
G=F_{1} \cup g_{2} F_{1} \cup \ldots \cup g_{p} F_{1},
$$

we can see that all elements of the coset $g_{i} F_{1}$ send the point $\mathrm{S}_{1}$ to the same point $\mathrm{S}_{i}=g_{i} F_{1} \mathrm{~S}_{1}=g_{i} \mathrm{~S}_{1}$. The set of points $\mathrm{S}_{i}$ is called the orbit. If we pick up any point $\mathrm{S}_{i}$ of the orbit and apply an element of the group $G$ to it, we obtain another point of the orbit. Indeed, $g \mathrm{~S}_{i}=g g_{i} \mathrm{~S}_{1}=g_{j} \mathrm{~S}_{1}=\mathrm{S}_{j}$. The stabilizer of a point $S_{i}$ is a group $F_{i}=g_{i} F_{1} g_{i}^{-1}$ conjugate to the group $F_{1}$. We consider now the first normalizer $N_{G}^{(1)}\left(F_{1}\right)$ of the subgroup $F_{1}$ in $G$. From coset resolutions

$$
G=N_{G}^{(1)}\left(F_{1}\right) \cup t_{2} N_{G}^{(1)}\left(F_{1}\right) \cup \ldots \cup t_{q} N_{G}^{(1)}\left(F_{1}\right)
$$

and

$$
N_{G}^{(1)}\left(F_{1}\right)=F_{1} \cup s_{2} F_{1} \cup \ldots \cup s_{r} F_{1}
$$

we obtain

$$
\begin{aligned}
G= & F_{1} \cup s_{2} F_{1} \cup \ldots s_{r} F_{1} \cup \\
& t_{2} F_{1} \cup t_{2} s_{2} F_{1} \cup \ldots t_{2} s_{r} F_{1} \cup \\
& \vdots \\
& t_{q} F_{1} \cup t_{q} s_{2} F_{1} \cup \ldots t_{q} s_{r} F_{1} .
\end{aligned}
$$

Changing the indexing of points, starting with $S_{1} \rightarrow S_{11}$, through $\mathrm{S}_{i j}=t_{i} s_{j} \mathrm{~S}_{1}$, we obtain that the orbit splits into $q$ subsets labelled by indices from the set $i=1,2, \ldots, q$ and each of these subsets contains $r$ points $\mathrm{S}_{i j}$ with fixed index $i$ and $j=1,2, \ldots, r$. The points with the same $i$ have the same stabilizer $F_{i}=g_{i} F_{1} g_{i}^{-1}$. The numbers $q$ and $r$ are indices of subgroups $q=\left[G: N_{G}^{(1)}\left(F_{1}\right)\right], \quad r=\left[N_{G}^{(1)}\left(F_{1}\right): F_{1}\right] \quad$ and $p=q r=\left[G: F_{1}\right]$.

If the set $\mathcal{S}$ is the space $V$ of tensors, then the states $\mathrm{S}_{i j}$ are described by tensors $\mathbf{x}_{i j}$ in this space. These tensors then characterize the individual domain states. It is suitable to use the typical spaces and variables for the description of these states.

Linear orbits and strata: If the set $\mathcal{S}$ on which the group $G$ acts is a linear space $V(n)$, then the elements are vectors $\mathbf{x} \in V(n)$. If $F_{1}$ is the stabilizer of a vector $\mathbf{x}_{11} \in V(n)$ then, using the same coset resolution as in the case of the set $\mathcal{S}$, we obtain an orbit of vectors $\mathbf{x}_{i j}=t_{i} s_{j} \mathbf{x}_{11}$ for which we use the name linear orbit. Since linear spaces are sets with some special properties, we can expect that linear orbits will also have some properties, characteristic of the linear action of the group $G$. Indeed, if vector $\mathbf{x}_{11}$ generates an orbit of vectors $\mathbf{x}_{i j}$, then every vector $a \mathbf{x}_{11}, a \neq 0$, generates an orbit of vectors $a \mathbf{x}_{i j}$. From this we can get the wrong impression that vectors with the same stabilizer $F_{1}$ form linear spaces. This, however, is not true, as we can already see by considering the trivial vector $\mathbf{x}=\mathbf{0}$ which belongs to every linear space and generates an orbit with one vector - itself. Also, if linearly independent vectors $\mathbf{x}$ and $\mathbf{y}$ have the same stabilizer $F_{1}$, then the stabilizer of their linear combination $a \mathbf{x}+b \mathbf{y}$ certainly contains $F_{1}$ but is not necessarily identical with it.

This means that, while all vectors of the stability space $V\left(F_{1}\right)$ are by definition invariant under the group $F_{1}$, the group $F_{1}$ is not necessarily the stabilizer of an arbitrary vector from $V\left(F_{1}\right)$. The set of all vectors of $V(n)$ that have $F_{1}$ as stabilizer will be called here the stratum of $F_{1}$ in $V(n)$. The stratum of $F_{1}$ in a certain space $V(n)$ can be empty. If it is not, then we find it as follows. We consider the stability space $V\left(F_{1}\right)$. This is never empty as it contains at least the trivial vector $\mathbf{x}=\mathbf{0}$. If the stability space is nontrivial, it is at least of dimension 1 . The stratum is then the set of all vectors of $V\left(F_{1}\right)$ with the exception of the trivial vector. If the stability space is of higher dimension, it may contain subspaces, which are stability spaces of supergroups of $F_{1}$. To get the stratum, we have to take all these stability spaces out of the space $V\left(F_{1}\right)$. Since these subspaces are of lower dimensions than $V\left(F_{1}\right)$, the stratum of $F_{1}$ in $V\left(F_{1}\right)$ is always a dense subset of $V\left(F_{1}\right)$. We shall use the more customary expression that vector $\mathbf{x}$ is a general vector of the stability space $V\left(F_{1}\right)$ as this expression is more usual in the literature. Indeed, the vectors that do not belong to the stratum can be considered as vectors of special symmetry.

Remark. This definition of stratum is different from its use in the mathematical literature where Stratum is defined as a collection of orbits of the same type. This Stratum is therefore the union of strata as defined here for the set of conjugate subgroups and it coincides with our concept of strata for a normal subgroup. If it is necessary to use both concepts, we suggest the use of a capital $S$ as the symbol for the original Stratum.

Kernels and epikernels: Let us now replace the set $\mathcal{S}$ by a typical carrier space $V_{\alpha}(G)$. If the corresponding $R$-irep $\chi_{\alpha}(G)$ is one-dimensional, $\operatorname{Ker} \chi_{\alpha}(G)=H_{\alpha}$, then the space $V_{\alpha}(G)$ contains only two types of orbits:

(i) the trivial vector $\mathbf{x}^{(\alpha)}=\mathbf{0}$, whose stabilizer under the action of $G$ is the group $G$ itself;

(ii) if $\mathbf{x}^{(\alpha)}=\mathbf{X}_{\alpha} \mathbf{e}^{(\alpha)}$, then the stabilizer of this vector is the halving subgroup $H_{\alpha}$, and, from coset resolution $G=H_{\alpha} \cup g_{2} H_{\alpha}$, we obtain the second vector of the orbit which is $g_{2} \mathbf{x}^{(\alpha)}=-\mathbf{x}^{(\alpha)}$.

Notice that the set of all vectors with stabilizer $H_{\alpha}$ is the whole space $V_{\alpha}(G)$ with the exception of the trivial vector. This set is the stratum of $H_{\alpha}$.

If the dimension of the $R$-irep is at least two, we have to analyse the structure of the space $V_{\alpha}(G)$ as follows.

(i) The stabilizer of the trivial vector $\mathbf{x}^{(\alpha)}=\mathbf{0}$ is again the group $G$ itself.

(ii) The stabilizer of any other vector $\mathbf{x}^{(\alpha)}$ is at least the group $H_{\alpha}=\operatorname{Ker} D_{R}^{(\alpha)}(G)$. The index of $H_{\alpha}$ is now higher than two and we find the orbit in the usual manner. If every vector of the space has this group for its stabilizer, then we take the trivial vector out and get the typical stratum of $H_{\alpha}$ in $V_{\alpha}(G)$. The stratum of the group $G$ always consists of the space $V_{1}(G)$ of its invariants, the stratum of $G$ in $V_{\alpha}(G), \alpha \neq 1$, contains only the trivial vector. In no other cases is the stratum a space.

(iii) There may, however, exist vectors of the space $V_{\alpha}(G)$, the stabilizers of which are greater than $H_{\alpha}$. Let us assume that such a vector $\mathbf{x}_{1}^{(\alpha)}$ exists. The stabilizer of this vector is called an epikernel of the $R$-irep $D_{R}^{(\alpha)}(G)$ and we denote it by $F_{\alpha 1}$. This group cannot be normal in $G$ because normal subgroups leave invariant either all vectors or only the trivial vector in each of 
Table 1

The typical stability spaces and epikernels of $R$-ireps for tetragonal groups isomorphic with $D_{4 z}-4_{z} 2_{x} 2_{x y}$.

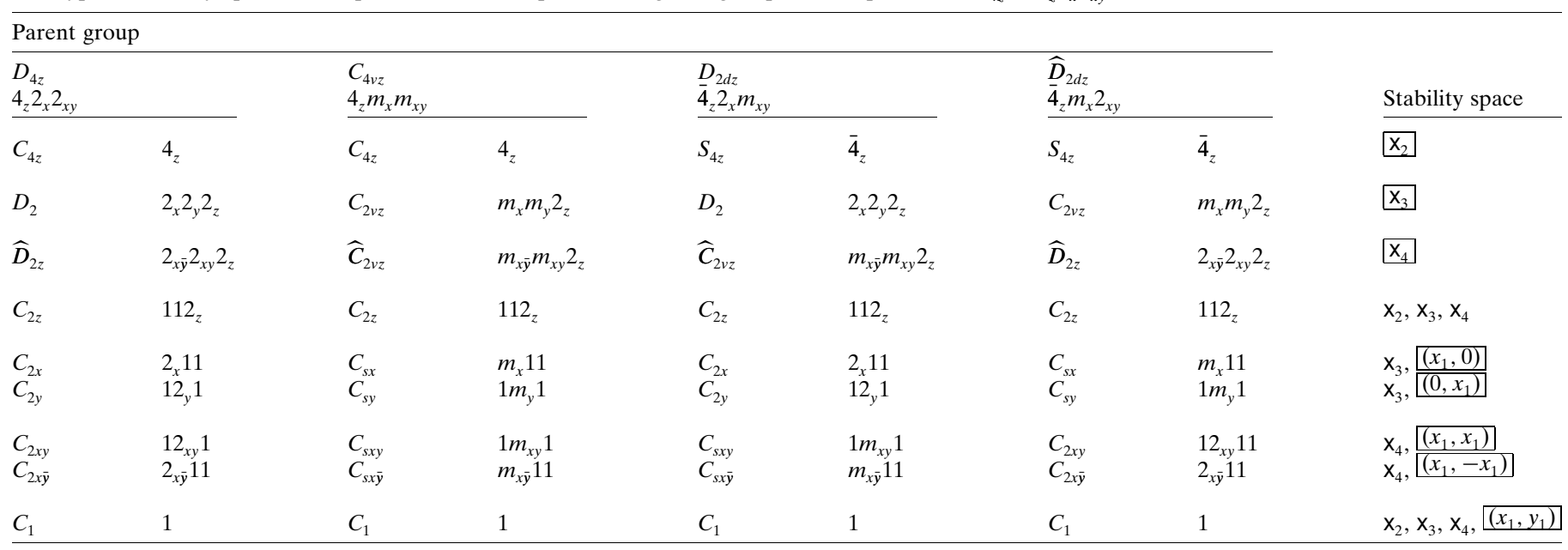

the spaces $V_{\alpha}(G)$. Epikernels therefore appear as sets of conjugate subgroups.

The stability space $V_{\alpha}\left(F_{\alpha 1}\right)$ is of the dimension $s_{\alpha}\left(F_{\alpha 1}\right)<d_{\alpha}$. If $s_{\alpha}\left(F_{\alpha 1}\right)>1$, which may happen only if $d_{\alpha}>2$, there may exist vectors of $V_{\alpha}\left(F_{\alpha 1}\right)$, the stabilizer of which is greater than $F_{\alpha 1}$. The dimension of the stability space of this stabilizer is smaller than $s_{\alpha}\left(F_{\alpha 1}\right)$.

The structure of the space $V_{\alpha}(G)$ can therefore be described as follows. A general vector of this space has the stabilizer $H_{\alpha}=\operatorname{Ker} \chi_{\alpha}(G)$. All vectors with exactly this stabilizer form the stratum of the group $H_{\alpha}$. This will be obtained if the trivial vector and vectors of stability spaces of epikernels are excluded from the space $V_{\alpha}(G)$.

Epikernels appear as sets of conjugate subgroups $F_{\alpha i}^{(u)}$, where the superscript $u$ labels different sets of conjugate subgroups and the index $i$ labels subgroups of the set. The intersections core $F_{\alpha}^{(u)}=\bigcap_{i} F_{\alpha i}^{(u)}=H_{\alpha}$ of all conjugate epikernels always result in the kernel. If one epikernel $F_{\alpha 1}^{(v)}$ is a subgroup of another epikernel $F_{\alpha 1}^{(u)}$, then the stability space $V_{\alpha}\left(F_{\alpha 1}^{(u)}\right)$ is a subspace of the stability space $V_{\alpha}\left(F_{\alpha 1}^{(v)}\right)$. The stratum of each epikernel is therefore obtained by excluding from its stability space the trivial vector and stability spaces of all epikernels which are supergroups of this epikernel.

In Tables 1 and 2 are presented typical stability spaces of subgroups of tetragonal point groups of the oriented Laue class $D_{4 z}-4_{z} 2_{x} 2_{x y}$ and epikernels of their ireps; Table 1 applies to those parent groups which are isomorphic to the group $D_{4 z}-4_{z} 2_{x} 2_{x y}$, Table 2 applies to the centrosymmetric group $D_{4 h z}-4_{z} / m_{z} m_{x} m_{x y}$. As a result of our choice of ireps and standard variables, it is easy to extend these results to all groups of the oriented Laue class $D_{4 z}-42_{z} 2_{x y}$ with the exception of the paramagnetic centrosymmetric group $D_{4 h z}^{\prime}-4_{z} / m_{z} m_{x} m_{x y} \cdot 1^{\prime}$, which must be considered separately.

The word typical means that the information is given in terms of typical variables which can always be replaced by corresponding tensor components by the use of tables of tensorial covariants. In the two tables, we list the subgroups by both Schoenflies and Hermann-Mauguin symbols and in the right-hand column we record the corresponding stability spaces for these subgroups. If the vector in this column is framed, then the subgroup is an epikernel of that irep to whose space the tensor belongs. Notice that we can present this information in one table for the four isomorphic groups not only because of their isomorphisms but also due to our standard choice of representations and typical variables.

The framed variables correspond to primary tensor parameters. These are defined as those parameters whose onset is the cause of the symmetry descent. The other parameters in the same line are then the consequence of this symmetry descent and we call them the secondary tensor parameters. Some subgroups are not epikernels of any of the ireps, so that no variable is framed. Such a symmetry descent needs tensor parameters from more than one irep and we can find which parameters if we look up the groups which are epikernels and whose intersection is the considered group. This boils down to a statement that each subgroup is either itself an epikernel or an intersection of epikernels. ${ }^{1}$ The difference between stability spaces and strata in these tables is simple. The typical variables must not be zero for a stratum while stability spaces also include vectors for which these variables vanish. The use of these tables is described below.

A philosophical digression: In connection with these tables, we may question the validity of the famous statement by Curie (1884a, p. 400, 1884b): 'C'est la dissymétrie qui crée le phénomène' ('dissymmetry creates the phenomenon'). From our consideration it seems more appropriate to say that the principal tensor parameters create (are responsible for) the symmetry descent or, in other words, are the cause of the descent. Though the secondary parameters may seem to be the consequence of the descent, it seems again more appropriate to explain their onset as a consequence of faint interaction (Kopský, 1979).

\footnotetext{
1 Analogous tables for other oriented Laue classes are available from the IUCr electronic archives (Reference: XO5008). Services for accessing these data are described at the back of the journal.
} 
Table 2

The typical stability spaces and epikernels of $R$-ireps for the group $D_{4 h z}-4_{z} / m_{z} m_{x} m_{x y}$.

\begin{tabular}{|c|c|c|}
\hline$C_{4 h z}$ & $4_{z} / m_{z}$ & $\mathrm{X}_{2}^{+}$ \\
\hline$D_{2 h}$ & $m_{x} m_{y} m_{z}$ & $\mathrm{X}_{3}^{+}$ \\
\hline$\widehat{D}_{2 h z}$ & $m_{x \bar{y}} m_{x y} m_{z}$ & $\mathrm{X}_{4}^{+}$ \\
\hline$D_{4 z}$ & $4 z 2_{x} 2_{x y}$ & $\mathrm{x}_{1}^{-}$ \\
\hline$C_{4 v z}$ & $4_{z} m_{x} m_{x y}$ & $\mathrm{x}_{2}^{-}$ \\
\hline$D_{2 d z}$ & $\overline{4}_{z} 2_{x} m_{x y}$ & $\mathrm{X}_{3}^{-}$ \\
\hline$\widehat{D}_{2 d z}$ & $\overline{4}_{z} m_{x} 2_{x y}$ & $\mathrm{x}_{4}^{-}$ \\
\hline$C_{2 h z}$ & $112_{z} / m_{z}$ & $\mathrm{x}_{2}^{+}, \mathrm{x}_{3}^{+}, \mathrm{x}_{4}^{+}$ \\
\hline$C_{4 z}$ & $4 z$ & $\mathrm{x}_{2}^{+}, \mathrm{x}_{1}^{-}, \mathrm{x}_{2}^{-}$ \\
\hline $\mathrm{D}_{2}$ & $22_{y} 2_{z}$ & $x_{3}^{+}, x_{1}^{-}, x_{3}^{-}$ \\
\hline$\widehat{D}_{2 z}$ & $2_{x \bar{y}} 2_{x y} 2_{z}$ & $\mathrm{x}_{4}^{+}, \mathrm{x}_{1}^{-}, \mathrm{x}_{4}^{-}$ \\
\hline$S_{4 z}$ & $\overline{4}_{z}$ & $\mathrm{x}_{2}^{+}, \mathrm{x}_{3}^{-}, \mathrm{x}_{4}^{-}$ \\
\hline$C_{2 v z}$ & $m_{x} m_{y} 2_{z}$ & $x_{2}^{-}, x_{3}^{+}, x_{4}^{-}$ \\
\hline$\widehat{C}_{2 v z}$ & $m_{x \bar{y}} m_{x y} 2_{z}$ & $\mathrm{x}_{2}^{-}, \mathrm{x}_{3}^{-}, \mathrm{x}_{4}^{+}$ \\
\hline$C_{2 z}$ & $112_{z}$ & $\mathrm{x}_{2}^{+}, \mathrm{x}_{3}^{+}, \mathrm{x}_{4}^{+}, \mathrm{x}_{1}^{-}, \mathrm{x}_{2}^{-}, \mathrm{x}_{3}^{-}, \mathrm{x}_{4}^{-}$ \\
\hline $\begin{array}{l}C_{2 h x} \\
C_{2 h y}\end{array}$ & $\begin{array}{l}2 x_{x} / m_{x} 11 \\
12_{y} / m_{y} 1\end{array}$ & $\begin{array}{l}\mathrm{x}_{3}^{+},\left(x_{1}^{+}, 0\right) \\
\mathrm{x}_{3}^{+},\left(0, x_{1}^{+}\right)\end{array}$ \\
\hline $\begin{array}{l}C_{2 h x y} \\
C_{2 h x \bar{y}}\end{array}$ & $\begin{array}{l}12_{x y} / m_{x y} 1 \\
2_{x \bar{y}} / m_{x \bar{y}} 11\end{array}$ & $\begin{array}{l}\mathbf{x}_{4}^{+},\left(x_{1}^{+}, x_{1}^{+}\right) \\
\mathbf{x}_{4}^{+},\left(x_{1}^{+},-x_{1}^{+}\right)\end{array}$ \\
\hline$C_{i}$ & $\overline{1}$ & $\mathrm{x}_{2}^{+}, \mathrm{x}_{3}^{+}, \mathrm{x}_{4}^{+},\left(x_{1}^{+}, y_{1}^{+}\right)$ \\
\hline $\begin{array}{l}C_{2 v x} \\
C_{2 v y}\end{array}$ & $\begin{array}{l}2{ }_{x} m_{y} m_{z} \\
m_{x} 2_{y} m_{z}\end{array}$ & $\begin{array}{l}\mathrm{x}_{3}^{+},\left(x_{1}^{-}, 0\right) \\
\mathrm{x}_{3}^{+},\left(0, x_{1}^{-}\right)\end{array}$ \\
\hline $\begin{array}{l}C_{2 v x y} \\
C_{2 v x \bar{y}}\end{array}$ & $\begin{array}{l}m_{x \bar{y}} 2_{x y} m_{z} \\
2_{x \bar{y}} m_{x y} m_{z}\end{array}$ & $\begin{array}{l}\mathrm{x}_{4}^{+},\left(x_{1}^{-}, x_{1}^{-}\right) \\
\mathrm{x}_{4}^{+},\left(x_{1}^{-},-x_{1}^{-}\right)\end{array}$ \\
\hline$C_{s z}$ & $11 m_{z}$ & $\mathrm{x}_{2}^{+}, \mathrm{x}_{3}^{+}, \mathrm{x}_{4}^{+},\left(x_{1}^{-}, y_{1}^{-}\right)$ \\
\hline $\begin{array}{l}C_{2 x} \\
C_{2 y}\end{array}$ & $\begin{array}{l}2 x 11 \\
12_{y} 1\end{array}$ & $\begin{array}{l}\mathrm{x}_{3}^{+}, \mathrm{x}_{1}^{-}, \mathrm{x}_{3}^{-},\left(x_{1}^{+}, 0\right),\left(x_{1}^{-}, 0\right) \\
\mathrm{x}_{3}^{+}, \mathrm{x}_{1}^{-}, \mathrm{x}_{3}^{-},\left(0, x_{1}^{+}\right),\left(0, x_{1}^{-}\right)\end{array}$ \\
\hline $\begin{array}{l}C_{2 x y} \\
C_{2 x \bar{y}}\end{array}$ & $\begin{array}{l}12_{x y} 1 \\
2_{x \bar{y}} 11\end{array}$ & $\begin{array}{l}\mathrm{x}_{4}^{+}, \mathrm{x}_{1}^{-}, \mathrm{x}_{4}^{-},\left(x_{1}^{+}, x_{1}^{+}\right),\left(x_{1}^{-}, x_{1}^{-}\right) \\
\mathrm{x}_{4}^{+}, \mathrm{x}_{1}^{-}, \mathbf{x}_{4}^{-},\left(x_{1}^{+},-x_{1}^{+}\right),\left(x_{1}^{-},-x_{1}^{-}\right)\end{array}$ \\
\hline $\begin{array}{l}C_{s x} \\
C_{s y}\end{array}$ & $\begin{array}{l}m_{x} 11 \\
1 m_{y} 1\end{array}$ & $\begin{array}{l}\mathrm{x}_{3}^{+}, \mathrm{x}_{2}^{-}, \mathrm{x}_{4}^{-},\left(x_{1}^{+}, 0\right),\left(0, x_{1}^{-}\right) \\
\mathrm{x}_{3}^{+}, \mathrm{x}_{2}^{-}, \mathrm{x}_{4}^{-},\left(0, x_{1}^{+}\right),\left(x_{1}^{-}, 0\right)\end{array}$ \\
\hline $\begin{array}{l}C_{s x y} \\
C_{s x \bar{y}}\end{array}$ & $\begin{array}{l}1 m_{x y} 1 \\
m_{x y} 11\end{array}$ & $\begin{array}{l}\mathrm{x}_{4}^{+}, \mathrm{x}_{2}^{-}, \mathrm{x}_{3}^{-},\left(x_{1}^{+}, x_{1}^{+}\right),\left(x_{1}^{-},-x_{1}^{-}\right) \\
\mathrm{x}_{4}^{+}, \mathrm{x}_{2}^{-}, \mathrm{x}_{3}^{-},\left(x_{1}^{+},-x_{1}^{+}\right),\left(x_{1}^{-}, x_{1}^{-}\right)\end{array}$ \\
\hline$C_{1}$ & $112_{z}$ & $\mathrm{x}_{2}^{+}, \mathrm{x}_{3}^{+}, \mathrm{x}_{4}^{+}, \mathrm{x}_{1}^{-}, \mathrm{x}_{2}^{-}, \mathrm{x}_{3}^{-}, \mathrm{x}_{4}^{-},\left(x_{1}^{+}, y_{1}^{+}\right),\left(x_{1}^{-}, y_{1}^{-}\right)$ \\
\hline
\end{tabular}

Remark. We consider it appropriate to make the following remark of historical and terminological character. Ascher (1977) introduced the term epikernel [see also Kobayashi \& Ascher (1977)] in connection with his study of the direct and inverse Landau problem and invented the Ker-core criterion, according to which the groups $F_{i}$ are epikernels of an irep of the class $\chi_{\alpha}(G)$ if and only if core $\left\{F_{i}\right\}=\bigcap_{i} F_{i}=$ $H_{\alpha}=\operatorname{Ker} \chi_{\alpha}(G)$. This criterion is more comfortable than the subduction (Birman, 1966) and chain subduction criteria (Jarič, 1981). Unfortunately, the terms little group or isotropy group crept into the literature instead of epikernel. In our opinion, this is taking jargon too far. A little linguistic analysis seems to be not out of place here. The older terms little group or isotropy group are now frequently replaced by the term stabilizer. The term was introduced in connection with the group action on any set. The stabilizer of a point of the set is then that subgroup which contains all those elements of the group which leave the point invariant. In this sense, an epikernel of an irep $D_{R}^{(\alpha)}(G)$ is a stabilizer (little group or isotropy group) of a certain vector of the carrier space $V_{\alpha}$ under the action of the group $G$ (it is by no means the stabilizer of its irep). In addition, the term little group (isotropy group) of an irep has its own meaning in the theory of representations of space groups, which is quite a different story.

\section{Exomorphic symmetry descents}

Let us consider two symmetry descents: $G \Downarrow F_{1}$ and $\widetilde{G} \Downarrow \widetilde{F}_{1}$. First we construct the intersections $H=\operatorname{core} F_{i}=\bigcap_{i} F_{i}$ and $\widetilde{H}=\operatorname{core} \widetilde{F}_{i}=\bigcap_{i} \widetilde{F}_{i}$ which are normal subgroups of $G$ and $\widetilde{G}$, respectively. If the factor groups $G / H$ and $\widetilde{G} / \widetilde{H}$ are isomorphic, then there exist homomorphisms $\sigma$ and $\widetilde{\sigma}$, with kernels $\operatorname{Ker} \sigma=H, \operatorname{Ker} \widetilde{\sigma}=\widetilde{H}$ which map the groups $G$ and $\widetilde{G}$ onto the same group $\mathcal{H}=\sigma(G)=\widetilde{\sigma}(\widetilde{G})$. If these homomorphisms also map the groups $F_{1}$ and $\widetilde{F}_{1}$ onto the same subgroup $\mathcal{F}_{1}=\sigma\left(F_{1}\right)=\widetilde{\sigma}\left(\widetilde{F}_{1}\right)$, then we say that the symmetry descents (group-subgroup relations) $G \Downarrow F_{1}$ and $\widetilde{G} \Downarrow \widetilde{F}_{1}$ are exomorphic or of the same exomorphic type. The following is a diagram of exomorphic relations.

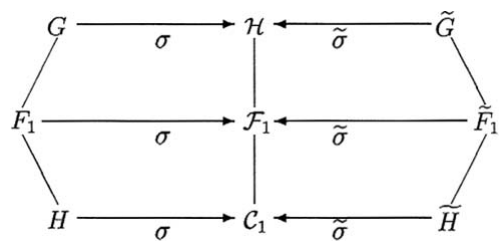

The exomorphism of symmetry descents has powerful consequences.

(i) There exists a one-to-one mapping of ireps $\alpha \longleftrightarrow \widetilde{\alpha}$ of $G$ and $\widetilde{G}$ engendered by ireps of the factor group $\mathcal{H}$. There also exists a one-to-one mapping of cosets $g_{i} F_{1} \longleftrightarrow \widetilde{g}_{i} \widetilde{F}_{i}$ such that elements of these cosets act in the same way on spaces of engendered ireps. Indeed, both cosets act in the same way as the element $\gamma_{i}$ of the factor group $\mathcal{H}$.

(ii) From this it follows that the stability spaces, strata and typical orbits have identical structures.

(iii) In addition, all polynomials in variables which belong to engendered ireps have the same transformation properties under the action of elements of corresponding cosets. 
The concept was originally introduced by Kopský (1978) for equitranslational phase transitions. In this context, we can say that the consideration of two exomorphic transitions is based on the same algebraic relations. In view of this, the classification of symmetry descents into exomorphic types facilitates systematic investigation. Thus in terms of crystallographic point groups we find 44 exomorphic types. Among them we find 28 cases when the subgroup is an epikernel of some irep, and in 5 cases out of this the subgroup is simultaneously an epikernel of two ireps. The number of distinct descents within classical crystallographic point groups is 212 , within magnetic crystallographic point groups it goes up to 1599 . In terms of equitranslational symmetry descents between ordinary or magnetic space groups, this number will go up into the thousands. The generalization of the concept is rather straightforward (Litvin et al., 1986).

Remark. The permutation representation of the group $G$ on cosets of the subgroup $F_{1}$ has also been used for derivation of colour groups. A later attempt to rename exomorphism as chromomorphism and a rather brutal attempt to change the usual terminology on the grounds of this fact belongs to the folklore of applications of group theory.

\section{The fine structure of a typical linear orbit}

We now consider again the typical stability space $V_{o}\left(F_{1}\right)$ of a subgroup $F_{1}$ of the group $G$. This space is a direct sum of the stability spaces $V_{\alpha}\left(F_{1}\right)$ of the group $F_{1}$ in the individual typical carrier spaces $V_{\alpha}$. We denote by $F_{\alpha 1}$ the stabilizer of a general vector $\mathbf{x}_{11}^{(\alpha)} \in V_{\alpha}\left(F_{1}\right)$. The stability space $V_{\alpha}\left(F_{1}\right)$ is identical with the stability space $V_{\alpha}\left(F_{\alpha 1}\right)$ and all stabilizers $F_{\alpha 1}$ contain the group $F_{1}$. We perform the coset resolution

$$
G=F_{\alpha 1} \cup g_{2}^{(\alpha)} F_{\alpha 1} \cup \ldots \cup g_{p_{\alpha}}^{(\alpha)} F_{\alpha 1}
$$

and the more detailed

$$
G=N_{G}^{(1)}\left(F_{\alpha 1}\right) \cup t_{2}^{(\alpha)} N_{G}^{(1)}\left(F_{\alpha 1}\right) \cup \ldots \cup t_{q_{\alpha}}^{(\alpha)} N_{G}^{(1)}\left(F_{\alpha 1}\right)
$$

and

$$
N_{G}^{(1)}\left(F_{\alpha 1}\right)=F_{\alpha 1} \cup s_{2}^{(\alpha)} F_{\alpha 1} \cup \ldots \cup s_{r_{\alpha}}^{(\alpha)} F_{\alpha 1}
$$

which combine into

$$
\begin{aligned}
G= & F_{\alpha 1} \cup s_{2}^{(\alpha)} F_{\alpha 1} \cup \ldots s_{r_{\alpha}}^{(\alpha)} F_{\alpha 1} \cup \\
& t_{2}^{(\alpha)} F_{\alpha 1} \cup t_{2}^{(\alpha)} s_{2}^{(\alpha)} F_{\alpha 1} \cup \ldots t_{2}^{(\alpha)} s_{r_{\alpha}}^{(\alpha)} F_{\alpha 1} \cup \\
& \vdots \\
& t_{q_{\alpha}}^{(\alpha)} F_{\alpha 1} \cup t_{q_{\alpha}}^{(\alpha)} s_{2}^{(\alpha)} F_{\alpha 1} \cup \ldots t_{q_{\alpha}}^{(\alpha)} s_{r_{\alpha}}^{(\alpha)} F_{\alpha 1}
\end{aligned}
$$

to obtain the orbit of vector $\mathbf{x}_{11}^{(\alpha)} \in V_{\alpha}\left(F_{\alpha 1}\right)=V_{\alpha}\left(F_{1}\right)$. This orbit contains $p_{\alpha}=q_{\alpha} r_{\alpha}$ vectors $\mathbf{x}_{i j}^{(\alpha)}=t_{i}^{(\alpha)} s_{j}^{(\alpha)} \mathbf{x}_{11}^{(\alpha)} \in V_{\alpha}\left(F_{\alpha i}\right)$ and splits into $q_{\alpha}$ subsets labelled by indices from the set $i=1,2, \ldots, q_{\alpha}$ and each of these subsets contains $r_{\alpha}$ vectors $\mathbf{x}_{i j}^{(\alpha)}$ with fixed index $i$ and index $j=1,2, \ldots, r_{\alpha}$. The vectors with the same $i$ have the same stabilizer $F_{\alpha i}=g_{i}^{(\alpha)} F_{\alpha 1} g_{i}^{(\alpha)-1}$. The numbers $q_{\alpha}$ and $r_{\alpha}$ are indices of subgroups: $q_{\alpha}=\left[G: N_{G}^{(1)}\left(F_{\alpha 1}\right)\right], \quad r_{\alpha}=\left[N_{G}^{(1)}\left(F_{\alpha 1}\right): F_{\alpha 1}\right]$ and $p_{\alpha}=q_{\alpha} r_{\alpha}=$ $\left[G: F_{\alpha 1}\right]$.

A vector $\mathbf{x}_{11}$ of the typical stability space $V_{o}\left(F_{1}\right)$ of the subgroup $F_{1}$ generates an orbit of $p=q r$ vectors $\mathbf{x}_{i j}=t_{i} s_{j} \mathbf{x}_{11}$ with stabilizers $F_{i}$, where $p=\left[G: F_{1}\right]=q r$, where $q=\left[G: N_{G}^{(1)}\left(F_{1}\right)\right]$ and $r=\left[N_{G}^{(1)}\left(F_{1}\right): F_{1}\right]$. This vector splits into components $\mathbf{x}_{11}^{(\alpha)}$ in the typical irreducible subspaces $V_{\alpha}$. Each of these components has its own stabilizer $F_{\alpha 1} \supseteq F_{1}$. Vectors $\mathbf{x}_{i j}=t_{i} s_{j} \mathbf{x}_{11}$ of the orbit also split into components $\mathbf{x}_{i j}^{(\alpha)}$. While all vectors $\mathbf{x}_{i j}$ of the typical orbit are distinct, the components $\mathbf{x}_{i j}^{(\alpha)}$ are also distinct only if $F_{\alpha 1}=F_{1}$. Notice that this means that these components are distinct only if $F_{1}$ is an epikernel of the irep $D_{R}^{(\alpha)}(G)$. On the other hand, if $F_{\alpha 1} \supset F_{1}$, the number $p_{\alpha}$ of distinct components is a divisor of the total number $p$ of vectors of the orbit; it is $p: p_{\alpha}=\left[F_{\alpha 1}: F_{1}\right]$.

If we label the components $\mathbf{x}_{i j}^{(\alpha)}$ by the original labels $i j$, then certain groups of these labels denote the same component. To label them uniquely, we use the coset resolutions associated with the stabilizer $F_{\alpha 1}$ which results in a certain unique labelling $\mathbf{x}_{i_{\alpha} j_{\alpha}}^{(\alpha)}$. Each label $i_{\alpha} j_{\alpha}$ then corresponds to $k_{\alpha}=p: p_{\alpha}$ labels $i j$. The set of vectors

$$
\mathbf{x}_{i j}=\left(\ldots, \mathbf{x}_{i_{\alpha} j_{\alpha}}^{(\alpha)}, \ldots, \mathbf{x}_{i_{\beta} j_{\beta}}^{(\beta)}, \ldots\right)
$$

will be called the typical linear orbit and its representation in terms of components in irreducible typical subspaces will be referred to as the fine structure of the typical linear orbit. Tables of these (under the less precise name fine domain structures and with different symbols of variables) were published a long time ago (Kopský, 1982a). The original classification of symmetry descents into exomorphic classes given in this work is used below in the examples.

In Table 3 are shown fine structures of typical orbits for a few of the symmetry descents from a particular parent group. The advantage of typical variables is clear if we recall that tables of tensorial covariants assign to each of these variables all covariant tensor components up to fourth order (and if extension to higher orders is necessary, we know how to find it).

To attempt a more precise terminology would be appropriate at this point to connect the abstract picture provided by group theory with real physical situations and also with their models. Formation of domains at structural phase transitions is a physically established and observed fact (actually, the concept of domains appears for the first time in connection with the theory of ferromagnetic materials where group theory is dwarfed, while demagnetization fields play the main role). Quite generally, a physical observation of a symmetry descent from the state $\mathcal{S}$ of parent symmetry $\mathcal{G}$ to some state $\mathcal{S}_{1}$ of low symmetry $\mathcal{F}_{1}$ implies that the state of the system may, in the absence of special external conditions, change equally likely to any of the states $\mathcal{S}_{i}=g_{i} \mathcal{S}_{1}$, where $g_{i}$ are coset representatives in the coset resolution

$$
\mathcal{G}=\mathcal{F}_{1} \cup g_{2} \mathcal{F}_{1} \cup \ldots \cup g_{p} \mathcal{F}_{1} .
$$


Table 3

Examples of the fine structure of typical orbits.

\begin{tabular}{llll}
\hline Symmetry descent: $D_{4 h z} \Downarrow D_{2 h}$ & & & \\
Exomorphic type: $1 . C_{2} \Downarrow C_{1}$ & & $D_{2 h}$ & \\
$D_{4 h z}$ & & $x_{3}^{+}$ & \\
$D_{2 h}$ & & & \\
\hline & & & \\
\hline Symmetry descent: $D_{4 h z} \Downarrow C_{4 z}$ & $C_{4 z}$ & $i C_{4 z}$ & 2 \\
Exomorphic type: $5 . D_{2} \Downarrow C_{1}$ & $\mathrm{x}_{2}^{+}$ & $\mathrm{x}_{2}^{+}$ & $-\mathrm{x}_{2}^{+}$ \\
$D_{4 h z}$ & $\mathrm{x}_{1}^{-}$ & $-\mathrm{x}_{1}^{-}$ & $\mathrm{x}_{1}^{-}$ \\
$C_{4 h z}$ & $\mathrm{x}_{2}^{-}$ & $-\mathrm{x}_{2}^{-}$ & $-\mathrm{x}_{2}^{-}$ \\
$D_{4 z}$ & & & $-\mathrm{x}_{2}^{+}$ \\
$C_{4 v z} C_{4 z}$ & $-\mathrm{x}_{1}^{-}$ \\
\hline
\end{tabular}

Symmetry descent: $D_{4 h z} \Downarrow\left(C_{2 h x}, C_{2 h y}\right)$
Exomorphic type: 7 b. $D_{4 z} \Downarrow\left(C_{2 x}, C_{2 y}\right)$

First normalizer $N_{D_{4 h z}}\left(C_{2 h x}\right)=N_{D_{4 h}, 2}\left(C_{2 h y}\right)=D_{2 h}=$ normal subgroup

$D_{4 h z}$

$\mathrm{D}_{2 h}$

${ }_{2} C_{2 h x}$

$4_{z} D_{2 h}$

${ }_{z}^{-1} C_{2 h x}$

$D_{2 h}$

$C_{2 h x}$
$\mathrm{X}_{3}^{+}$

$C_{2 h i}$

$\left(x_{1}^{+}, 0\right)$

$\mathrm{x}_{3}^{+}$

$\left(-x_{1}^{+}, 0\right)$

$C_{2 h y}$
$-\mathbf{X}_{3}^{+}$

$\left(0, x_{1}^{+}\right)$

$-\mathrm{x}_{3}^{+}$

$\left(0,-x_{1}^{+}\right)$

Symmetry descent: $D_{4 h z} \Downarrow C_{s z}$

Exomorphic type: 7 a. $D_{4 z} \Downarrow C_{1}$

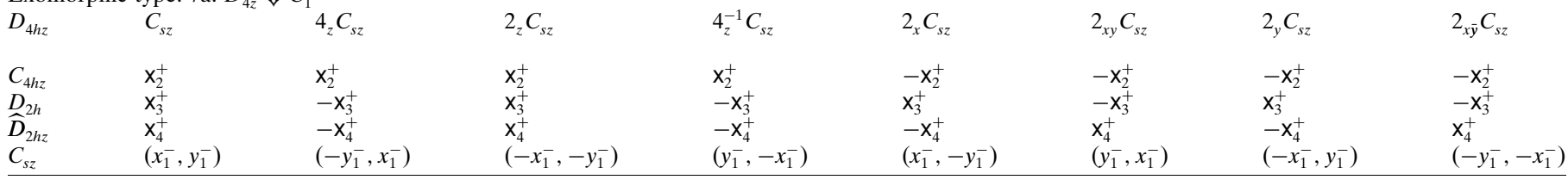

Symmetry descent: $D_{4 h z} \Downarrow\left(C_{2 x}, C_{2 y}\right)$

Exomorphic type: $14 b$. itself

First normalizer $N_{D_{4 h z}}\left(C_{2 x}\right)=N_{D_{4 h z}}\left(C_{2 y}\right)=D_{2 h}=$ normal subgroup

$D_{4 h z}$

$D_{2 h}$
$C_{2 x}$

$i C_{2 x}$

${ }_{z} C_{2 x}$

$m_{z} C_{2 x}$

${ }_{2} D_{2 h}$

${ }^{4} C_{2 y}$

$\overline{4}_{z} C_{2 x}$

$4_{z}^{-1} C_{2 x}$

$\overline{4}_{z}^{-1} C_{2 x}$

$\begin{array}{ll}D_{2 h} & \mathrm{x}_{3}^{+} \\ D_{4} & \mathrm{x}_{1}^{-} \\ D_{2 d z} & \mathrm{x}_{3}^{-} \\ C_{2 h i} & \left(x_{1}^{+}, 0\right) \\ C_{2 v i} & \left(x_{1}^{-}, 0\right)\end{array}$

$\mathrm{x}_{3}^{+}$
$-\mathrm{x}_{1}^{-}$
$-\mathrm{x}_{3}^{-}$
$\left(x_{1}^{+}, 0\right)$
$\left(-x_{1}^{-}, 0\right)$

$\mathrm{x}_{3}^{+}$
$\mathrm{x}_{1}^{-}$
$\mathrm{x}_{3}^{-}$
$\left(-x_{1}^{+}, 0\right)$
$\left(-x_{1}^{-}, 0\right)$

$\mathrm{x}_{3}^{+}$

$-x_{1}^{-}$

$-x_{1}$
$-x_{3}^{-}$
$\left(-x_{1}^{+}, 0\right)$

$\left(-x_{1}^{+}, 0\right)$

$\left(x_{1}^{-}, 0\right)$

$-\mathrm{x}_{3}^{+}$
$\mathrm{x}_{1}^{-}$
$-\mathrm{x}_{3}^{-}$
$\left(0, x_{1}^{+}\right)$
$\left(0, x_{1}^{-}\right)$

$-\mathrm{x}_{3}^{+}$
$-\mathrm{x}_{1}^{-}$
$\mathrm{x}_{3}^{-}$
$\left(0, x_{1}^{+}\right)$
$\left(0,-x_{1}^{-}\right)$

$-x_{3}^{+}$

$\mathrm{x}_{1}^{-}$

$-\mathrm{x}_{3}^{-}$

$\left(0,-x_{1}^{+}\right)$

$\left(0,-x_{1}^{-}\right)$

$-\mathrm{x}_{1}^{-}$

$\left(0,-x_{1}^{+}\right)$

$\left(0, x_{1}^{-}\right)$

$\left(0,-x_{1}^{-}\right)$

$\left(0, x_{1}^{-}\right)$

The states $\mathcal{S}_{i}$ are called the domain states. It is usually said that the domain states $\mathcal{S}_{i}$ are symmetrically equivalent. It is probably more precise to say that all relationships of the original state $\mathcal{S}$ to any of the states $\mathcal{S}_{i}$ are mutually geometrically equivalent and hence physically equivalent. Indeed, any element of $g_{i} \mathcal{F}_{1}$ leaves the original state $\mathcal{S}$ invariant and the change of the relationship between the state $\mathcal{S}$ and $\mathcal{S}_{1}$ to the relationship between the state $\mathcal{S}$ and $\mathcal{S}_{j}$ is obtained by applying an element of $g_{j} F_{1}$ to both states. The effect of this operation is the rotation and, in full structural considerations, also the possible space shift of the situation in physical space.

The state of the crystal at the transition usually does not change to any particular state $\mathcal{S}_{i}$. The crystal splits instead into regions, called domains, and the state in each of the domains is one of the domain states. In addition, if the transition is ferroelastic, the domain states coincide with those predicted by coset resolution only in the vicinity of the transitions point (socalled parent clamping approximation). Ferroelastic phase transitions play a special role in the theory of domains for the following reason: the domain states are eventually realized in the individual domains. The structures of a crystal, which correspond to various domain states, are identical up to their orientation and location in the space. Domains are therefore regions of physical space occupied by such states and there exist interfaces (boundaries) between these regions. Group theory predicts the equivalence of the number of domain states equal to the index $\left[\mathcal{G}: \mathcal{F}_{1}\right]$ of the low symmetry to the parent symmetry. In reality, only two domains can meet at an interface of a certain orientation and location. From grouptheoretical analysis, we may predict possible mutual orientations and locations of pairs of respective domain states and continue the analysis for any orientation and location of an 
interface. However, in the case of ferroelastic phase transitions, there exist physical conditions on the existence of interfaces - the boundary between two domains should be stress free. As a result, the mutual orientation and location of domain states coincides with that predicted by group theory only when spontaneous distortions of the structure are small.

In the examples $D_{4 h z} \Downarrow D_{2 h}, D_{4 h z} \Downarrow\left(C_{2 h x}, C_{2 h y}\right)$ and $D_{4 h z} \Downarrow C_{s z}$, we can observe that each domain state corresponds to a distinct vector in one of the typical spaces. There are the variables or vectors $\mathrm{x}_{3}^{+},\left(x_{1}^{+}, 0\right)$ and $\left(x_{1}^{-}, y_{1}^{-}\right)$in the first domain state, which change from one domain to the other, so that all domains are characterized by distinct vectors. In the case of descents $D_{4 h z} \Downarrow D_{2 h}, D_{4 h z} \Downarrow C_{s z}$, we need to take vectors of two ireps to distinguish all domain states.

\section{Labelling of covariants and conversion equations}

Substituting actual tensor components for typical variables in the tables of the fine structure of typical orbits, we obtain as complete a description of all domain states in terms of their tensor properties as the tables of tensorial covariants provide. If this turns out not to be sufficient, we can always extend the tables of tensorial covariants to higher ranks.

However, if we inspect tables of covariants or if we try an actual substitution, we shall find that typical variables are substituted by covariant tensor components which are frequently linear combinations of Cartesian components. It is therefore necessary to convert this information into the Cartesian frame of reference. The situation is analogous to the consideration of invariant tensor forms. Invariants are frequently linear combinations of Cartesian components and, as a result, we obtain an invariant tensor in the Cartesian frame which has more allowed components than the number of independent invariants. In this case, there appear relations between the Cartesian components which reduce the number of independent components to the correct one.

The effect in ferroic transitions is that Cartesian components are not necessarily the principal parameters and there appear increments to these components which are again not independent. To resolve this problem, we introduce the method of labelling the covariants and of conversion equations (Kopský, 2001a,b,c), which was later used by Janovec \& Kopský (2003).

The method is illustrated in Table 4 for the tetragonal group $D_{4 z}$. Table 4(a) defines the labels of covariants as linear combinations of Cartesian components and Table 4(b) gives the Cartesian components expressed as linear combinations of covariant components. The principles of notation are explained below. It is our opinion that it would be useful to standardize the labels of covariants.

To each group there are assigned two tables. The first table, called 'Labelling of covariants' assigns numerical labels to linearly independent $D^{(\alpha)}(G)$ covariants in the case when more than one covariant of a given tensor to this irep exists. We shall illustrate it for the case of tensor A. One-dimensional covariants, including invariants, are denoted by sans-serif letter $A$ with two indices, the first of which is the numerical label of an irep, the second is the number of the covariant. If only one covariant of the type exists, we drop the second index. Thus, $A_{1}$ means an invariant, $A_{3}$ means a $\chi_{3}(G)$ covariant but $A_{1,1}, A_{1,2}, A_{1,3}$ mean the first, second and third invariants and $\mathrm{A}_{3,1}, \mathrm{~A}_{3,2}$ mean the first and second $\chi_{3}(G)$ covariants. The two-dimensional $D^{(1)}(G)$ covariants are expressed $\quad$ as $\quad \mathbf{A}_{1}^{(1)}=\left(A_{1 x, 1}, A_{1 y, 1}\right), \quad \mathbf{A}_{2}^{(1)}=\left(A_{1 x, 2}, A_{1 y, 2}\right)$, $\mathbf{A}_{3}^{(1)}=\left(A_{1 x, 3}, A_{1 y, 3}\right)$ and so on if more than three linearly independent $D^{(1)}(G)$ covariants exist while $\mathbf{A}^{(1)}=\left(A_{1 x}, A_{1 y}\right)$ signals that there is no other $D^{(1)}(G)$ covariant.

The second table bears the title 'Conversion equations' and contains Cartesian tensor components expressed in terms of the covariant components. If all non-invariant components are set to zero, we get the tensor form, invariant under the group $G$. By comparison with the main tables, we can see which covariant components onset at each transition from the parent group $G$. Thus these components can be described as the tensor parameters of the transition. Conversion equations are a convenient platform to launch the detailed investigation of domain pairs and domain walls. The results of such an investigation in a form which an experimentalist can directly use are in preparation as a continuation of this work.

Example: Using the conversion Table 4 for the group $D_{4 z}-4 z 2_{x} 2_{x y}$ and Table 1 of stability spaces and epikernels, find how the Cartesian components of the piezoelectric tensor d change as we descend with the symmetry (on various paths) to trivial symmetry $C_{1}-1$.

First we take into account that tensor $\mathbf{d}$ transforms in this group in the same way as tensor $\mathbf{A}$ for which labelling of covariants and conversion equations are listed. We just replace the characters $A$ and A by $d$ and d.

In the group $D_{4 z}-4_{z} 2_{x} 2_{x y}$, we find that only two components are nonvanishing: $d_{14}=-d_{25}=\frac{1}{2} \mathrm{~d}_{1}$ and equal to one invariant with opposite signs.

In the group $C_{4 z}-4_{z}$, variable $\mathrm{x}_{2}$ onsets to which there correspond Cartesian components $d_{31}=d_{32}=\frac{1}{2} \mathrm{~d}_{2,1}$, $d_{15}=d_{24}=\frac{1}{2} \mathbf{d}_{2,2}$ and $d_{33}=\mathrm{d}_{2,3}$.

To the group $D_{2}-2 x 2_{y} 2_{z}$ there corresponds variable $\mathrm{x}_{3}$ which indicates onset of the component $d_{36}=\mathrm{d}_{3,2}$. The covariant $\mathrm{d}_{3,1}$ contributes to already allowed components by $\Delta d_{14}=\Delta d_{25}=\frac{1}{2} \mathrm{~d}_{3,1}$. Owing to this increment, the components $d_{14}$ and $d_{25}$ are now independent.

To the group $\widehat{D}_{2}-2_{x \bar{y}} 2_{x y} 2_{z}$ there corresponds variable $\mathrm{x}_{4}$ which contributes to Cartesian components by $d_{31}=-d_{32}=\frac{1}{2} \mathrm{~d}_{4,1}$ and $d_{15}=-d_{24}=\frac{1}{2} \mathrm{~d}_{4,2}$. These Cartesian components are not independent because of the orientation of this group.

All three variables $x_{2}, x_{3}, x_{4}$ are invariant under the group $C_{2 z}$ and hence all components $d_{14}, d_{25}, d_{31}, d_{32}, d_{15}$ and $d_{24}$ are independent and can be expressed through two consecutive increments as seen from conversion equations.

In the group $C_{2 x}-2$, we obtain again the increments $\Delta d_{14}=\Delta d_{25}=\frac{1}{2} \mathrm{~d}_{3,1}$ to components already allowed in the parent group. In addition, the first component $\left(x_{1}, 0\right)$ is now allowed, to which there corresponds an onset of tensor components: $d_{11}=d_{1 x, 1}, d_{13}=d_{1 x, 3}, d_{35}=d_{1 x, 5}, d_{12}=d_{1 x, 2}$ and $d_{26}=d_{1 x, 4}$. 
Table 4

Labelling of covariants and conversion equations: Group $4_{z} 2_{x} 2_{x y}-\left(D_{4 z}\right)$.

(a) Labelling of covariants

\begin{tabular}{|c|c|c|c|}
\hline $\begin{array}{l}\mathrm{u}_{1,1}=u_{1}+u_{2} \\
\mathrm{u}_{1,2}=u_{3}\end{array}$ & & $\begin{array}{l}\mathrm{u}_{3}=u_{1}-u_{2} \\
\mathbf{u}^{11}=\left(u_{1 x}, u_{1 y}\right)=\left(u_{4},-u_{5}\right)\end{array}$ & $\mathrm{u}_{4}=u_{6}$ \\
\hline $\mathrm{A}_{1}=A_{14}-A_{25}$ & $\begin{array}{l}\mathrm{A}_{2,1}=A_{31}+A_{32} \\
\mathrm{~A}_{2,2}=A_{15}+A_{24} \\
\mathrm{~A}_{2,3}=A_{33}\end{array}$ & $\begin{array}{l}\mathrm{A}_{3,1}=A_{14}+A_{25} \\
\mathrm{~A}_{3,2}=A_{36}\end{array}$ & $\begin{array}{l}\mathrm{A}_{4,1}=A_{31}-A_{32} \\
\mathrm{~A}_{4,2}=A_{15}-A_{24}\end{array}$ \\
\hline $\begin{array}{l}\mathbf{A}_{11}^{(1)}=\left(A_{1 x, 1}, A_{1 y, 1}\right)=\left(A_{11}, A_{22}\right) \\
\mathbf{A}_{2}^{(1)}=\left(A_{1 x, 2}, A_{1 y, 2}\right)=\left(A_{12}, A_{21}\right)\end{array}$ & & $\begin{array}{l}\mathbf{A}_{3}^{(1)}=\left(A_{1 x, 3}, A_{1 y, 3}\right)=\left(A_{13}, A_{23}\right) \\
\mathbf{A}_{4}^{(1)}=\left(A_{1 x, 4}, A_{1 y, 4}\right)=\left(A_{26}, A_{16}\right) \\
\mathbf{A}_{5}^{(1)}=\left(A_{1 x, 5}, A_{1 y, 5}\right)=\left(A_{35}, A_{34}\right)\end{array}$ & \\
\hline
\end{tabular}

(b) Conversion equations

\begin{tabular}{|c|c|c|c|}
\hline $\begin{array}{l}u_{1}=\frac{1}{2}\left(\mathrm{u}_{1,1}+\mathrm{u}_{3}\right) \\
u_{4}=u_{1 x}\end{array}$ & $\begin{array}{l}u_{2}=\frac{1}{2}\left(\mathrm{u}_{1,1}-\mathrm{u}_{3}\right) \\
u_{5}=-u_{1 y}\end{array}$ & $\begin{array}{l}u_{3}=\mathrm{u}_{1,2} \\
u_{6}=\mathrm{u}_{4}\end{array}$ & \\
\hline $\begin{array}{l}A_{11}=A_{1 x, 1} \\
A_{13}=A_{1 x, 3} \\
A_{35}=A_{1 x, 5}\end{array}$ & $\begin{array}{l}A_{22}=A_{1 y, 1} \\
A_{23}=A_{1 y, 3} \\
A_{34}=A_{1 y, 5} \\
A_{14}=\frac{1}{2}\left(\mathrm{~A}_{1}+\mathrm{A}_{3,1}\right) \\
A_{31}=\frac{1}{2}\left(\mathrm{~A}_{2,1}+\mathrm{A}_{4,1}\right) \\
A_{15}=\frac{1}{2}\left(\mathrm{~A}_{2,2}+\mathrm{A}_{4,2}\right)\end{array}$ & $\begin{array}{l}A_{12}=A_{1 x, 2} \\
A_{26}=A_{1 x, 4} \\
A_{33}=\mathrm{A}_{2,3} \\
A_{25}=\frac{1}{2}\left(-\mathrm{A}_{1}+\mathrm{A}_{3,1}\right) \\
A_{32}=\frac{1}{2}\left(\mathrm{~A}_{2,1}-\mathrm{A}_{4,1}\right) \\
A_{24}=\frac{1}{2}\left(\mathrm{~A}_{2,2}-\mathrm{A}_{4,2}\right)\end{array}$ & $\begin{array}{l}A_{21}=A_{1 y, 2} \\
A_{16}=A_{1 y, 4} \\
A_{36}=\mathrm{A}_{3,2}\end{array}$ \\
\hline$s_{14}=s_{1 x, 1}$ & $s_{25}=-s_{1 y, 1}$ & $s_{24}=s_{1 x, 2}$ & $s_{15}=-s_{1 y, 2}$ \\
\hline$s_{34}=s_{1 x, 3}$ & $s_{35}=-s_{1 y, 3}$ & $s_{56}=s_{1 x, 4}$ & $s_{46}=-s_{1 y, 4}$ \\
\hline$q_{13}=\frac{1}{2}\left(\mathrm{q}_{1,1}+\mathrm{q}_{3,1}\right)$ & $q_{23}=\frac{1}{2}\left(\mathrm{q}_{1,1}-\mathrm{q}_{3,1}\right)$ & $q_{16}=\frac{1}{2}\left(\mathrm{q}_{2,1}+\mathrm{q}_{4,1}\right)$ & $q_{26}=\frac{1}{2}\left(-\mathrm{q}_{2,1}+\mathrm{q}_{4,1}\right)$ \\
\hline & $q_{45}=\mathrm{q}_{2,2}$ & $q_{12}=\mathrm{q}_{3,2}$ & $q_{36}=\mathrm{q}_{4,2}$ \\
\hline$q_{14}=q_{1 x, 1}$ & $q_{25}=-q_{1 y, 1}$ & $q_{24}=q_{1 x, 2}$ & $q_{15}=-q_{1 y, 2}$ \\
\hline$q_{34}=q_{1 x, 3}$ & $q_{35}=-q_{1 y, 3}$ & $q_{56}=q_{1 x, 4}$ & $q_{46}=-q_{1 y, 4}$ \\
\hline
\end{tabular}

In the group $C_{2 y}-2_{y}$, we obtain again the increments $\Delta d_{14}=\Delta d_{25}=\frac{1}{2} \mathrm{~d}_{3,1}$ to components already allowed in the parent group. In addition, the second component $\left(0, x_{1}\right)$ is now allowed, to which there corresponds an onset of tensor components: $d_{22}=d_{1 y, 1}, d_{23}=d_{1 y, 3}, d_{34}=d_{1 y, 5}, d_{21}=d_{1 y, 2}$ and $d_{16}=d_{1 y, 4}$. The magnitude of these components must be the same as that of corresponding components in the group $C_{2 x}-2_{x}$, because the state is actually another domain state of the same descent.

In the groups $C_{2 x y}-2_{x y}$ and $C_{2 x \bar{y}}-2_{x \bar{y}}$, we obtain Cartesian components $d_{31}=-d_{32}=\frac{1}{2} \mathrm{~d}_{4,1} \quad$ and $\quad d_{15}=-d_{24}=\frac{1}{2} \mathrm{~d}_{4,2}$. Vector $\left(x_{1}, x_{1}\right)$ indicates that in the group $C_{2 x y}-2_{x y}$ we obtain $d_{11}=d_{22}, d_{13}=d_{23}, d_{35}=d_{34}, d_{12}=d_{21}$ and $d_{26}=d_{16}$, while, in the other group $C_{2 x \bar{y}}-2_{x \bar{y}}, d_{11}=-d_{22}, d_{13}=-d_{23}$, $d_{35}=-d_{34}, d_{12}=-d_{21}$ and $d_{26}=-d_{16}$.

We have to check now that all these components taken together are all components of tensor $\mathbf{d}$ as it should be in the trivial group $C_{1}-1$.

Conversion equations are not necessary for parent groups up to $m_{x} m_{y} m_{z}-D_{2 h}$ because Cartesian components are themselves relative invariants (this is, however, due to the choice of group orientations). Labelling of covariants and solution of conversion equations for other tensors and for other groups can be performed by rewriting results for groups of proper rotations with the use of Opechowski's magic relations (Kopský, 2006a). 
Table 5

Twinning groups for pair of domain states in symmetry descents from the parent group $D_{4 z}-42_{z} 2_{x y}$.

\begin{tabular}{|c|c|}
\hline Parent group & Twinning group \\
\hline $\begin{array}{l}4_{z}\left(C_{4 z}\right) \\
2_{x}\end{array}$ & $4_{z} 2_{x}^{\star} 2_{x y}^{\star}\left[D_{4 z}^{\star}\left(C_{4 z}\right)\right]$ \\
\hline $\begin{array}{l}2_{x} 2_{y} 2_{z}\left(D_{2}\right) \\
2_{x \bar{y}}\end{array}$ & $4_{z}^{\star} 2_{x} 2_{x y}^{\star}\left[D_{4 z}^{\star}\left(D_{2}\right)\right]$ \\
\hline $\begin{array}{l}2_{x \bar{y}} 2_{x y} 2_{z}\left(\widehat{D}_{2 z}\right) \\
2_{x}\end{array}$ & $4_{z}^{\star} 2_{x}^{\star} 2_{x y}\left[D_{4 z}^{\star}\left(\widehat{D}_{2 z}\right)\right]$ \\
\hline $\begin{array}{l}2_{z}\left(C_{2 z}\right) \\
2_{x} \\
2_{x y} \\
4_{z}\end{array}$ & $\begin{array}{l}2_{x}^{\star} 2_{y}^{\star} 2_{z}\left[D_{2}^{\star}\left(C_{2 z}\right)\right] \\
2_{x \bar{y}}^{\star} 2_{x y}^{\star} 2_{z}\left[D_{2}^{\star}\left(C_{2 z}\right)\right] \\
4_{z}^{\star}\left[C_{4 z}^{\star}\left(C_{2 z}\right)\right]\end{array}$ \\
\hline $\begin{array}{l}2_{x}\left(C_{2 x}\right) \\
2_{z} \\
2_{x y} \\
2_{x \bar{y}}\end{array}$ & $\begin{array}{l}2_{x} 2_{y}^{\star} 2_{z}^{\star}\left[D_{2}^{\star}\left(C_{2 x}\right)\right] \\
4_{z} 2_{x} 2_{x y}\left(D_{4 z}\right)\end{array}$ \\
\hline $\begin{array}{l}2_{x y}\left(C_{2 x y}\right) \\
2_{z} \\
2_{x} \\
2_{y}\end{array}$ & $\begin{array}{l}2_{x \bar{y}}^{\star} 2_{x y} 2_{z}^{\star}\left[\widehat{D}_{2 z}^{\star}\left(C_{2 x y}\right)\right] \\
4_{z} 2_{x} 2_{x y}\left(D_{4 z}\right)\end{array}$ \\
\hline $\begin{array}{l}1\left(C_{1}\right) \\
2_{z} \\
2_{x} \\
2_{y} \\
2_{x y} \\
2_{x \bar{y}} \\
4_{z} \\
4_{z}^{3}\end{array}$ & $\begin{array}{l}2_{z}^{\star}\left[C_{2 z}^{\star}\left(C_{1}\right)\right] \\
2_{x}^{\star}\left[C_{2 x}^{\star}\left(C_{1}\right)\right] \\
2_{y}^{\star}\left[C_{2 y}^{\star}\left(C_{1}\right)\right] \\
2_{x y}^{\star}\left[C_{2 x y}^{\star}\left(C_{1}\right)\right] \\
2_{x \bar{y}}^{\star}\left[C_{2 x y}^{\star}\left(C_{1}\right)\right] \\
4_{z}\left(C_{4 z}\right)\end{array}$ \\
\hline
\end{tabular}

\section{The twinning group and completely transposable pairs of domain states}

The original tables of the fine structure of domain states (Kopský, 1982a) have now been revised because some of the original numerical labels of ireps were changed to a more systematic labelling. With their use and with the use of conversion equations, we are able to describe all domain states by desirable tensors. This might be useful, for example, in averaging domain states of engineered multidomain structures with certain weights as well as in the determination of tensor distinction of domain states.

In consideration of domain walls or twin boundaries, we consider only pairs of domain states because only two domains meet at the wall. Here we are interested in the tensor distinction of pairs of domain states. Domain walls, pairs of domain states and their tensor distinction were extensively studied by Janovec and others (Janovec et al., 1992, 1993, 1994, 1995; Fuksa \& Janovec, 1995; Fuksa, 1997). In this connection, the concept of the embracing group or fundamental group of dichromatic pattern (Shubnikov \& Kopcik, 1974; Pond \& Vlachavas, 1983; Wadhawan, 2000) introduced originally as a bicrystallographic construction has been given the better name of the twinning group ( $c f$. Litvin \& Kopský, 1997, on magnetic twins; Janovec, Hahn \& Klapper, 2003; Janovec \& Přívratská, 2003).
Table 6

Twinning groups for pair of domain states in symmetry descents of low index from the parent group $O_{h}-m \overline{3} m$.

\begin{tabular}{|c|c|}
\hline Parent group & Twinning group \\
\hline $\begin{array}{l}m \overline{3}\left(T_{h}\right) \\
m_{x y}\end{array}$ & $m \overline{3} m^{\star}\left[O_{h}^{\star}\left(T_{h}\right)\right]$ \\
\hline $\begin{array}{l}432(O) \\
i\end{array}$ & $m^{\star} \overline{3}^{\star} m^{\star}\left[O_{h}^{\star}(O)\right]$ \\
\hline $\begin{array}{l}\overline{4} 3 m\left(T_{d}\right) \\
i\end{array}$ & $m^{\star} \overline{3}^{\star} m\left[O_{h}^{\star}\left(T_{d}\right)\right]$ \\
\hline $\begin{array}{l}23(T) \\
i \\
2_{x y} \\
m_{x y}\end{array}$ & $\begin{array}{l}m^{\star} \overline{3}^{\star}\left[T_{h}^{\star}(T)\right] \\
4^{\star} 32\left[O^{\star}(T)\right] \\
\overline{4}^{\star} 3 m^{\star}\left[T_{d}^{\star}(T)\right]\end{array}$ \\
\hline $\begin{array}{l}4_{z} / m_{z} m_{x} m_{x y}\left(D_{4 h z}\right) \\
m_{y z} \\
m_{z x}\end{array}$ & $m \overline{3} m\left(O_{h}\right)$ \\
\hline $\begin{array}{l}m_{x} m_{y} m_{z}\left(D_{2 h}\right) \\
m_{x y} \\
m_{y z} \\
m_{z x} \\
3_{p} \\
3_{p}^{2}\end{array}$ & $\begin{array}{l}4_{z}^{\star} / m_{z} m_{x} m_{x y}^{\star}\left[D_{4 h z}^{\star}\left(D_{2 h}\right)\right] \\
4_{x}^{\star} / m_{x} m_{y} m_{y z}^{\star}\left[D_{4 h x}^{\star}\left(D_{2 h}\right)\right] \\
4_{y}^{\star} / m_{y} m_{z} m_{z x}^{\star}\left[D_{4 h y}^{\star}\left(D_{2 h}\right)\right] \\
m \overline{3}\left(T_{h}\right)\end{array}$ \\
\hline $\begin{array}{l}4_{z} / m_{z}\left(C_{4 h z}\right) \\
m_{x} \\
m_{z x} \\
m_{z \bar{x}} \\
m_{y z} \\
m_{y \bar{z}}\end{array}$ & $\begin{array}{l}4_{z} / m_{z} m_{x}^{\star} m_{x y}^{\star}\left[D_{4 h z}^{\star}\left(C_{4 h z}\right)\right] \\
m 3 m\left(O_{h}\right)\end{array}$ \\
\hline
\end{tabular}

The twinning group: To study the distinction of the tensorial properties of a pair of domain states $\left(\mathrm{S}_{1}, \mathrm{~S}_{2}\right)$, it is frequently not necessary to know the symmetry descent $G \Downarrow F$ or the whole set of domain states. It is sufficient to find an element $g$ which sends the state $S_{1}$ into the state $S_{2}=g S_{1}$. The twinning group $T=T_{12}=T\left(\mathrm{~S}_{1}, \mathrm{~S}_{2}\right)=\left(F_{1}, g\right)$ is defined as the group generated by the symmetry of the first state and an element $g$ which sends it to the second state.

The next two Tables 5 and 6 refer to parent groups $D_{4 z}-42_{z} 2_{x y}$ and $O_{h}-m \overline{3} m$. Separated by blank lines are symmetry descents to subgroups of these two groups (in the second case only the descents of low index are given). Under each of the subgroups are listed representatives of cosets in the coset resolution of the parent group. The elements of each coset send the first domain state to another distinct domain state. The twinning group is given in the right column.

We shall consider here only the case of so-called completely transposable pairs of domain states which have the property that from $\mathrm{S}_{2}=g^{\star} \mathrm{S}_{1}$ follows $\mathrm{S}_{1}=g^{\star} \mathrm{S}_{2}$. In this case, the two states have the same symmetry which is a halving subgroup of the twinning group $T$. This leads to a notation in the style of magnetic point groups: some generators in the symbol of the twinning group are starred in the Hermann-Mauguin symbols, in Schoenflies symbols the star is attached to the symbol of $T$ and the subgroup $F_{1}$ is specified in parentheses. All elements of the coset $g^{\star} F_{1}$ transpose the domain states $\mathrm{S}_{1}$ and $\mathrm{S}_{2}$. The tensor properties of the two states are the same for those 
tensor components that are invariant under the group $T$, they differ in sign for those components that correspond to symmetry descent $T \Downarrow F_{1}$. This descent may not be the actual symmetry descent $G \Downarrow F_{1}$ at which the two domain states arise and tensor components by which the states differ may belong to an irep of $G$ which is not one-dimensional. On the other hand, this means that an experiment that leads to such a pair of states does not necessarily imply the actual symmetry descent. If $G \neq T$, which implies $T \subset G$, this may need the knowledge of a wider set of domain states.

Although the concept of the twinning group is useful for classification purposes and in cases of twinning, it is not quite necessary in the determination of the tensor distinction of domain states where tables of fine structure of domain states in conjunction with the 'Main Tables' of tensor parameters of the first domain state in ferroic symmetry descents [Kopský (2001a), and GI $\star$ KoBo-1 (Kopský \& Boček (2003)] provide more complete information. Thus in Table 3.4.3.4. of section 3.4 of International Tables for Crystallography (2003, Vol. D, pp. 478-479) are given only the numbers of components of tensors $\varepsilon$ (enantiomorphism), $\mathbf{P}$ (polarization), $g$ (gyrotropy), $d$ (piezoelectricity), $A$ (electrogyrotropy), $s$ (elastic stiffness) and $Q$ (elastooptics) in which pairs of domain states of completely transposable domain pairs differ. In an example below, we show the difference between the full analysis and a shortcut provided by the twinning group.

Example: We consider the transition $O_{h} \Downarrow\left\{C_{4 h z}, C_{4 h x}, C_{4 h y}\right\}$ or, in Hermann-Mauguin notation, $m \overline{3} m \Downarrow\left\{4_{z} / m_{z}, 4_{x} / m_{x}, 4_{y} / m_{y}\right\}$. The principal parameters for the first domain state $S_{1}$ with symmetry $C_{4 h z}\left(4_{z} / m_{z}\right)$ can be found on page 381 of the paper by Kopský (2001a) as covariant tensor components corresponding to variable $z_{1}^{+}$. These are $A_{33}, A_{32}+A_{31}, A_{24}+A_{15}, s_{26}-s_{16}, Q_{26}-Q_{16}$, $Q_{62}-Q_{61}, Q_{45}-Q_{54}=q_{45}$. Secondary tensor parameters correspond to typical vector $\left(x_{3}^{+}, 0\right)$. Twinning operation $m_{x}$ sends the vector $\left(0,0, z_{1}^{+}\right)$to $\left(0,0,-z_{1}^{+}\right)$and leaves the vector $\left(x_{3}^{+}, 0\right)$ intact. The pair of domain states $\left(\mathrm{S}_{1}, \mathrm{~S}_{2}\right)$, where $\mathrm{S}_{2}=m_{x} \mathrm{~S}_{1}$, is completely transposable and the corresponding twinning group is $4_{z} / m_{z} m_{x}^{\star} m_{x y}^{\star}\left[D_{4 h z}^{\star}\left(C_{4 h z}\right]\right.$. On page 165 of the same work, we find tensor parameters in which the two domain states differ as $A_{33}, A_{32}+A_{31}, A_{24}+A_{15}, s_{26}-s_{16}$, $Q_{26}-Q_{16}, Q_{62}-Q_{61}, Q_{45}-Q_{54}=q_{45}$, i.e. we get the same result as before without the use of the twinning group. If we look up the subduction tables, which were given together with fine structures of domain states, we find that the variable $z_{1}^{+}$in the group $O_{h}-m \overline{3} m$ subduces the variable $\mathrm{X}_{2}^{+}$which is to be replaced by these tensor parameters in the group $C_{4 h z}-4_{z} / m_{z}$.

With the use of conversion equations, we find that the transition is accompanied by an onset of Cartesian components $A_{33}, A_{32}=-A_{31}, A_{24}=-A_{15}, s_{26}=s_{16}, Q_{26}=Q_{16}$, $Q_{26}=Q_{16}$ and $q_{45}$ which change their sign if we go from the domain state $S_{1}$ to the state $S_{2}$. Thus we can obtain explicitly those tensor parameters in which the domain states differ as well with the use of the twinning group as without it. On the other hand, the table on page 381 of the cited work provides as easily the information about the other two pairs of domain states which correspond to typical variables $x_{1}^{+}$and $y_{1}^{+}$and to symmetries $C_{4 h x}-4_{x} / m_{x}$ and $C_{4 h y}-4_{y} / m_{y}$. The first pair is distinguished by tensor components $A_{11}, A_{13}=-A_{12}$, $A_{35}=-A_{26}, s_{34}=s_{24}, Q_{34}=Q_{24}, Q_{43}=Q_{42}$ and $q_{56}$, the second by components $A_{22}, A_{21}=-A_{23}, \quad A_{16}=-A_{34}$, $s_{15}=s_{35}, Q_{15}=Q_{35}, Q_{51}=Q_{53}$ and $q_{64}$. Thus we obtain a complete description of the distinction of the six domains with covariant as well as Cartesian components of tensors $A, s$ and $Q(q$ is the antisymmetric part of $Q$ ).

In addition, we can also obtain the information about the secondary parameters which correspond to variables, transforming by the irep $D^{(3+)}\left(O_{h}\right)$, where the domain states are characterized by tensor parameters corresponding to states $\quad\left(x_{3}^{+}, 0\right), \quad\left(-a x_{3}^{+}-b y_{3}^{+}, b x_{3}^{+}-a y_{3}^{+}\right) \quad$ and $\left(-a x_{3}^{+}+b y_{3}^{+},-b x_{3}^{+}-a y_{3}^{+}\right)$. These states correspond to symmetries $D_{4 h z}-4_{z} / m_{z} m_{x} m_{x y}, \quad D_{4 h x}-4_{x} / m_{x} m_{y} m_{y z}$ and $D_{4 h y}-4_{y} / m_{y} m_{z} m_{z x}$. With the use of conversion equations, we can find the distinction of pairs of domain states that belong to different choices of these symmetries in terms of those components of tensors $u, A, s$ and $Q$ which appear in the block, corresponding to the first state with the symmetry $D_{4 h z}-4_{z} / m_{z} m_{x} m_{x y}$.

Thus the twinning group is only of auxiliary value in cases when we need to consider only a certain pair of domain states. Complete description of domain states in terms of tensors up to fourth rank may be obtained from the 'Main tables' [Kopský (2001a) or GI KoBo-1 (Kopský \& Boček, 2003)] and from tables of the fine structure of domain states. An experienced user can even bypass the use of the latter tables because the transformations of the typical variables do not go beyond the action of symmetry operations on two- or threedimensional spaces. The essential points of an analysis of tensor properties of domain states are the decomposition of the tensor into tensorial covariants and the conversion equations.

\section{Discussion}

It's a long way to Tipperrary! Indeed, let us recall the steps of this version of tensor calculus and its outcome. We begin with meticulous specification of magnetic point groups and their isomorphisms. Precise specification of typical variables is a central point of systemization while the Clebsch-Gordan products represent the background for calculation of tensorial covariants or rather of the decomposition of tensors into these covariants. The choice of isomorphisms and of symbols for typical variables together with Opechowski's magic relations maximally simplify this task and cut down the tedious part of calculations to a minimum.

The knowledge of tensorial covariants is itself a starting point for the investigation of ferroic phase transitions, especially for the consideration of tensor properties of individual domain states which is necessary in modelling the properties of multidomain systems. The typical variables also prove to be useful in this application. With their use in tables of stability spaces and epikernels, we establish the quantities that characterize the first domain and in tables of fine structure of 
domain states we find complete information about these states. Up to orthorhombic parent groups, we do not need such a sophisticated scheme because the ireps are one-dimensional and tensorial covariants (relative invariants in these cases) coincide with Cartesian components. Starting with tetragonal parent groups, we meet, however, the difference between transition parameters and Cartesian tensor components. There are two options for resolving this discrepancy. We may obtain the results in terms of Cartesian tensor components using the procedure of labelling the covariants with subsequent transformation of results to Cartesian system through conversion equations. It would be, however, also possible to express the experimental results directly in terms of covariant components.

One of the problems of the practical use not only of this theory but also of the tables by experimentalists is the necessity of learning a certain minimal pensum of group theory such as given in this work. Tables are of no use if one does not know what information they contain. Let me, however, give an example that shows that learning at least the basic principles is worth the time. A recognized experimentalist asked me recently whether it is in order if he uses the relation $s_{i^{\prime} j^{\prime} k^{\prime} l^{\prime}}=c_{i i^{\prime}} c_{j j^{\prime}} c_{k k^{\prime}} c_{l l^{\prime}} s_{i j k l}$ where $c_{i i^{\prime}}$ are the matrices of vector transformation to calculate the changes of tensor $s$ from one domain state to another. Like the method of projection operators to find tensorial bases, this is theoretically correct. But in practice? With 21 components of the tensor $s$ and up to 48 domain states? Would it not be more productive to spend the time necessary for such error-prone calculations by learning the basic principles of the theory of irreducible representations? Indeed, the principle behind our system is the same as used in spectroscopy. Using covariant tensor components we split the space of dimension 21 to subspaces whose maximal dimension is 3 .

All information necessary to find tensor characteristics of individual domain states up to tensors of fourth rank and for the ordinary point groups is contained in the monograph by Kopský (2001a) and in the software GI KoBo-1 (Kopský \& Boček, 2003). Explicit tabulation of analogous results for magnetic point groups and magnetic properties in printed form is out of the question as well as the tabulation of the final results because of the volume required. On the other hand, the volume of basic tables from which desired information can be derived is relatively small and algorithms for its derivation rather transparent. We are preparing an extension of the software $G I \star K o B o-1$ (Kopský \& Boček, 2003) which will provide explicit results for magnetic point groups and magnetic properties with the hope that it will be helpful to experimentalists.

This work is part of project AVO 10100520 of the Czech Academy and has been supported by grant No. 202/04/0992 of the Czech Grant Agency. Thanks are due to Professor M. Nespolo, who gave the author the opportunity to present the results in lectures at the Summer School of the MathCryst group in Nancy, and to the French Embassy in Prague (Service de coopération et d'action culturelle) for generous support of local expenses as well as to my friend Professor D. B. Litvin who took care of the accommodation.

\section{References}

Ascher, E. (1977). J. Phys. C: Solid State Phys. 10, 1365-1377.

Birman, J. L. (1966). Phys. Rev. Lett. 17, 1216-1219.

Curie, P. (1884a). Bull. Soc. Fr. Minéral. 7, 418-457.

Curie, P. (1884b). J. Phys. (Paris), 3, 393-415.

Fuksa, J. (1997). Ferroelectrics, 204, 135-155.

Fuksa, J. \& Janovec, V. (1995). Ferroelectrics, 172, 343-350.

International Tables for Crystallography (2003). Vol. D, Physical Properties of Crystals, edited by A. Authier. Dordrecht: Kluwer Academic Publishers.

Janovec, V. (1972). Czech. J. Phys. B22, 974-994.

Janovec, V., Hahn, T. \& Klapper, H. (2003). International Tables for Crystallography, Vol. D, section 3.2, Twinning and Domain Structures, pp. 377-392. Dordrecht: Kluwer Academic Publishers.

Janovec, V. \& Kopský, V. (2003). International Tables for Crystallography, Vol. D, section 3.1.3, Equitranslational Phase Transitions. Property Tensors at Ferroic Phase Transitions, pp. 350-361. Dordrecht: Kluwer Academic Publishers.

Janovec, V., Litvin, D. B. \& Fuksa, J. (1995). Ferroelectrics, 172, 351-359.

Janovec, V., Litvin, D. B. \& Richterová, L. (1994). Ferroelectrics, 157, $75-80$.

Janovec, V. \& Přívratská, J. (2003). International Tables for Crystallography, Vol. D, section 3.4, Domain Structures, pp. 449-502. Dordrecht: Kluwer Academic Publishers.

Janovec, V., Richterová, L. \& Litvin, D. B. (1992). Ferroelectrics, 126, 287-292.

Janovec, V., Richterová, L. \& Litvin, D. B. (1993). Ferroelectrics, 140, 95-100.

Jarič, V. (1981). Phys. Rev. 23, 3460-3463.

Kobayashi, J. \& Ascher, E. (1977). J. Phys. C: Solid State Phys. 10, 1349-1364.

Kopský, V. (1978). Phys. Lett. A69, 82-84.

Kopský, V. (1979). J. Phys. A: Math. Gen. 12, L291-L294.

Kopský, V. (1982a). Group Lattices, Subduction of Bases \& Fine Domain Structures for Magnetic Point Groups. Prague: Academia.

Kopský, V. (1982b). Czech. J. Phys. B33, 485-509.

Kopský, V. (1982c). Czech. J. Phys. B33, 720-744.

Kopský, V. (1982d). Czech. J. Phys. B33, 845-869.

Kopský, V. (2001a). Phase Transit. 73, Nos. 1-2 (Special issue), pp. $1-422$.

Kopský, V. (2001b). Ferroelectrics, 252, 21-30.

Kopský, V. (2001c). Ferroelectrics, 252, 51-58.

Kopský, V. (2006a). Z. Kristallogr. 221, 51-62.

Kopský, V. (2006b). Acta Cryst. A62, 47-64.

Kopský, V. \& Boček, P. (2003). GI $\star$ KoBo-1 (Group Informatics, release 1). CD ROM Supplement to International Tables for Crystallography, Vol. D.

Landau, L. D. (1937). In Collected Papers of L. D. Landau (1967), edited by D. Ter Haar, pp. 193-216. New York: Gordon and Breach. [JETP, 7, pp. 19 and 627 (in Russian); Phys. Z. Sowjet. 11, pp. 26 and 545 (in German).]

Litvin, D. B., Fuksa, J. \& Kopský, V. (1986). J. Math. Phys. 27, 661-667.

Litvin, D. B. \& Kopský, V. (1997). Ferroelectrics, 204, 217-223.

Lyubarskii, G. Ya. (1960). The Applications of Group Theory in Physics. Oxford: Pergamon Press.

Pond, R. C. \& Vlachavas, V. A. (1983). Proc. R. Soc. London Ser. A, 386, 95-143.

Shubnikov, A. V. \& Kopcik, V. A. (1974). Symmetry in Science and Art. New York: Plenum Press.

Wadhawan, H. (2000). Introduction to Ferroic Materials. Amsterdam: Gordon and Breach. 